\title{
Review on Microscopic Detection Methods of Concrete under High
}

\section{Temperature}

\author{
Dongfu Zhao ${ }^{1,2, a}$, Huixuan Liu ${ }^{2, b, *}$ \\ ${ }^{1}$ Beijing Collaborative Innovation Center of Energy Conservation and Emissions Reduction \\ Technology, Beijing University of Civil Engineer and Architecture, Beijing 100044; \\ ${ }^{2}$ Beijing University of Civil Engineer and Architecture, Beijing 100044 \\ a zhaodongfu@bucea.edu.cn, ${ }^{b}$ mushroom0620@163.com
}

\begin{abstract}
Based on a lot of literatures at home and abroad, five kinds of detection methods, namely, scanning electron microscope test, X-ray diffraction test, ultrasonic test, microhardness test and mercury intrusion test are summarized by reviewing the studies of micro- and meso- structures of concrete after high temperature, and the problems that need to be solved between the current high temperature history of concrete and the microscopic detection method are discussed.
\end{abstract}

Keywords: concrete, high temperature, microscopic detection methods

\section{Introduction}

The structure of the building suffers from damage at different levels after the fire. By lots of researches have been done on the concrete, it's found that the changes of mechanical properties of concrete after fire are basically caused by microstructural changes. And the durability mechanism of concrete depends mainly on its internal chemical structure, work performance and the ability of the material itself to resist erosion, which is the result of multi-factor interaction. Therefore, the study of the microstructure of concrete materials has a very important role and significance. In this paper, five methods to study the micro- and meso-structures of concrete are summarized, and the problems that need to be solved between the current high temperature history of concrete and the microscopic detection method are discussed.

\section{Microscopic detection methods}

Many experts at home and abroad have optimized nondestructive testing methods of concrete at room temperature, so as to find detection and evaluation methods appropriately for fire-damaged concrete structures. Through the summary, microscopic detection methods that commonly used include five types, which are scanning electron microscope test, X-ray diffraction test, ultrasonic test, microhardness test and mercury intrusion test.

\subsection{Scanning electron microscope test}

By using scanning electron microscope (SEM), Punek and Branko [1] reserved the microcracks' development of concrete at different temperatures, and found that degradation of concrete at high temperature is one of the key factors, furthermore, high temperature plays a very important role in the development of different types of aggregates and microstructures. Liu Xian, Liu Yixiang and so on [2, 3] found that microsructures are obviously different at different temperatures. The higher temperature, the more porous the cement and the structure in the interfacial transition zone, the 
more loose the cement slurry, while the less dense the hydration products, C-S-H gel network structure gradually broken and disappeared, $\mathrm{AFt}$ and $\mathrm{Ca}(\mathrm{OH})_{2}$ gradually decomposition, the weaker the bonding power between aggregate and cement paste, the greater the degree of crack expansion. With the use of scanning electron microscopy, combined with the data obtained by ultrasonic testing, You Zuokai et al. [4] came up with a method to deduce the maximum temperature of the concrete in the actual project.

With the use of scanning electron microscope, researchers found that in the fire and other high temperature environment, the concrete structure will produce cement hydration products and cement mineral phase changes.

\subsection{X-ray diffraction test}

Fares et al. [5] studied the microstructure and physical properties of self-repairing concrete and the compressive strength after high temperature by X-ray diffraction. Lv Tianqi, Zhao Guofan et al. [6] used X-ray diffraction to find that the material composition of the concrete material varied at different temperatures, then they sliced the concrete structure, and observed the phase composition and the characteristics at different temperatures using an electron microscope to determine the fire temperature. Sun Zongquan [7] prepared the non-admixture concrete, by analyzing the XRD pattern, he found that the hydration reaction generated a large number of fiber-shape C-S-H and ettringite crystals for the needle-like, some large size and complete crystal hydroxide appeared. Yu Hongfa [8] discovered that the main crystalline phase in the cementite was $\mathrm{SiO}_{2}$, and a small amount of hydrated calcium aluminate, calcium hydroxide, $\mathrm{CaCO}_{3}$ and $\mathrm{CaSO}_{4} \cdot 2 \mathrm{H}_{2} \mathrm{O}$. The analysis results by DTA-TG are basically corresponding to that by XRD, expect to find more C-S-H gel.

$\mathrm{XRD}$ test and scanning electron microscopy test can be confirmed between the hydration products observed, and the relevant characteristics can be used as a sign of concrete temperature history.

\subsection{Ultrasonic test}

Zheng Sheng'e [9] used ultrasonic testing technology to analysis the relationship between the speed of sound, strength and other factors, and gave a quantitative assessment of the damage degree of concrete caused by high temperature. The assessment shows that the method of quantitative evaluation of concrete post fire by ultrasonic method is feasible, taking sound velocity into consider can reduce or eliminate the impact of most of the influencing factors (such as cement usage, water-cement ratio, coarse aggregate type and particle size, etc.), but its impact on the water content, temperature difference effect is small, the results of these two evaluations should be amended. Yujiang Tao [10] proposed an algorithm to monitor the depth of damage using ultrasonic detection method, the improved hyperbolic model and the parabolic model is established. Zhang Keqiang, Yang Bo et al. [11] put the $\mathrm{C} 20$ concrete block at $50^{\circ} \mathrm{C}$ high temperature and placed for different times, after natural cooling, its compressive strength standard value were tested by ultrasonic and rebound comprehensive method, the relationship of sound speed, rebound value and strength of C20 concrete post fire was obtained, and the strength curve was given.

It is found that the total porosity of high strength concrete increases with the increase of temperature. When the temperature is low, the total porosity increases slowly. This rule is helpful to judge the temperature process.

\subsection{Microhardness test}

Liu Haifeng [12] researched the hydration of cementitious system and the interface of aggregate-cement by interfacial microhardness test and scanning electron microscopy. Zhong Xiaolin [13] proposed a kind of high strength and wear resistant concrete, and analyzed the effect of this kind of external admixture on the concrete structure by microhardness test method. Finally, it 
explained that the external admixture increased the strength and wear resistance of concrete. Zhao Dongfu et al. [14] studied the microstructural changes of the materials subjected to different temperatures and different constant temperature with microhardness test. The physical and chemical changes of the high strength concrete materials and the microstructure changes caused by the high temperature were analyzed.

The microhardness test can detect the damage of the relevant strength and the decrease of the mechanical properties after the specimen being subjected to the fire, but the relationship between the microstructure parameter, the high temperature history and the stress damage can't be revealed.

\subsection{Mercury intrusion test}

Liu Xian [2] analyzed the physical and chemical changes of high performance concrete materials and the microcosmic results caused by the high temperature process through the mercury pressure measuring method. It was found that the main reason for the loss of material quality during the heating process was dehydration and various decomposition reactions. Yu Hongfa [8] found that gel pores, transitional pores, pores and big pores account for $57.71 \%, 23.05 \%, 6.76 \%$ and $12.48 \%$, respectively, with the largest pore diameter of $52 \mathrm{~nm}$. It can be seen that the pore structure of high performance concrete is dominated by gel pores with pore size $<10 \mathrm{~nm}$. Liu Hongbin [15] studied the fractal characteristics of pore structures and pore fractures in activated powder concrete (RPC) at high temperature by mercury intrusion porosity method. The fractal dimension of the RPC and the change of the temperature in the effective aperture range between the pores and the transition pore were calculated and analyzed.

These results show that high temperature plays an important role in the expansion of microcracks in concrete and reflects the change of aggregate type and microstructure of concrete.

\section{Conclusions}

The fire damage of concrete structure is extremely complex, and the accurate and rapid detection of the damage degree of the structural structure is a practical problem that needs to be solved urgently. However, in the actual detection, there are the following deficiencies:

1)Detection methods are varieties, but there is not enough evidence between the various research methods and the results;

2)There is little research on non-destructive testing of concrete after fire;

3)Existing research failed to well link the micro- and meso-structural changes and the temperature process that concrete experienced.

Based on the existing micro-means and the micro-means assessment methods, the maximum temperature concrete experienced during the fire can be obtained. What's more, the damage of the macroscopic mechanical properties of the concrete can be gotten by relevant literature formulas, which will provide the basis for post-disaster assessment. It will be the main direction of the future study of micro- and meso- structure of concrete.

\section{Acknowledgments}

The present work was supported by the National Natural Science Foundation of China (Project code: 51378045), and Open Research Fund Program of Engineering Structure and New Materials of Beijing University Engineering Research Center (Beijing University of Civil Engineering and Architecture). The authors gratefully acknowledge the mentioned financial support. 


\section{References}

[1] Punek, Branko. Study of microstructure and linear changes in fire-resistant concrete, Gradevinar. 48(1996) 241.

[2] Liu Xian, Yuan Yong, Ye Guang, Geert De Schutter. Study on pore structure evolution of high performance concrete with elevated temperatures, Journal of Tongji University: Natural Science. 36 (2008) 1473-1478.

[3] Liu Yixiang, Hu Jianguo, Hou Shuang. SEM analysis of concrete heated on varied temperature, Fire Safety Science. 15 (2005) 80-83.

[4] You Zuokai, Zhao Dongfu. Research on concrete microstructure evolution and the performance of RC beam after high temperature, Beijing: Beijing University of Civil Engineering and Architecture, 2013.

[5] Fares H S, Noumowe A, Cousture A. High temperature behaviour of self-consolidating concrete: microstructure and physicochemical properties, Cement and Concrete Research. 40 (2010) 488-496.

[6] Lv Tianqi, Zhao Guofan, Lin Zhishen. Microscopic analysis of static concrete after high temperature. Journal of Building Materials. 6 (2003) 135-141.

[7] Sun Zonquan, Liu Bin. Experimental study on corrosion resistance and microstructure hydration properties of high performance fair-faced concrete, Highway Engineering. 39 (2014) 309-313.

[8] Yu Hongfa, Liu Junlong, Zhang Yunsheng, Sun Wei, Li Meidan. Microstructure and durability forming mechanism of high performance concrete, Journal of Nanjing University of Aeronautics \& Astronautics. 39 (2007) 240-243.

[9] Zhen Sheng'e, Yang Yanke. Damage assessment of concrete after ultrasonic method for high temperature, Railway Engineering. 6 (1993) 10-14.

[10] Yu Jiangtao. Experimental and theoretical research on damage assessment of reinforced concrete member after fire, Shanghai: Tongji University. 2007.

[11] Zhang Keqiang, Yang Bo, Cheng Lihong. Nondestructive testing of C20 concrete strength after high temperature, Nondestructive Testing. 24 (2002) 339-340.

[12] Liu Haifeng, Gao Jianming, Wang Bian, Zhou Dianshen, Xu Xiyuan. Experimental study on microscopic performance of concrete mixed with slag, China concrete and cement products. 6 (2003) 16-18.

[13] Zhong Xiaolin, Sun Yuesheng, Zhong Chaoming, Zhu Zemin. The performances and hydration mechanism of high strength and abrasive-resistance concrete admixture, Concrete. 186(2005)81-87. [14]Zhao Dongfu, Liu Mei. Experimental study on residual strength and nondestructive testing of high strength concrete after high temperature. Journal of Building Structures. s12 (2015) 365-372.

[15] Liu Hongbin, Ju Yang, Sun Huafei, Liu Jinhui, Tian Kaipei, Wang Li, Ge Zhishun. Investigation on fractal characteristic of reactive powder concrete pore structure subject to high temperature, Journal of China Coal Society. 38 (2013) 1583-1588.

[16] Luo Fei, Zhu Jie. Research progress on microstructure of high performance concrete, Nonferrous Metals Engineering \& Research. 37 (2016) 88-90.

[17] Wang Xiaodong, Zhang Peng, Zhao Tiejun. Summary of testing method for chloride ion permeability in concrete, Engineering design and construction. 37 (2005) 25-29.

[18] Emmanuel,V. R. Annerel,Luc R. Taerwe. Basic approach for the diagnosis of concrete after fire exposure, Structural Fire Engineering. 1 (2010) 135-144. 